\title{
Intraductal Ultrasonography as a Local Assessment Before Magnetic Compression Anastomosis for Obstructed Choledochojejunostomy
}

\author{
Hideaki Kawabata ${ }^{\mathrm{a}, \mathrm{b}}$, Misuzu Hitomi ${ }^{\mathrm{a}}$, Naonori Inoue ${ }^{\mathrm{a}}$, Yukino Kawakatsu ${ }^{\mathrm{a}}$, \\ Yuji Okazaki ${ }^{\mathrm{a}}$, Masatoshi Miyata ${ }^{\mathrm{a}}$
}

\begin{abstract}
Magnetic compression anastomosis (MCA) has been developed as a non-surgical alternative treatment for biliary obstruction without serious complications. A 70-year-old woman who had undergone pancreaticoduodenectomy with modified Child reconstruction for pancreatic head cancer suffered from obstructed choledochojejunostomy with no recurrent findings 4 months after the operation. Cholangiography using the percutaneous transhepatic cholangiographic drainage (PTCD) and fluoroscopy revealed complete obstruction of the upper common bile duct, and the length of the obstruction was $7 \mathrm{~mm}$. Intraductal ultrasonography (IDUS) showed fibrous heterogenous hyperechoic appearance without fluid collection, vessels or foreign bodies at the site of the obstruction. We performed choledochojejunostomy using the MCA technique. One magnet was inserted into the obstruction of the hepatic side through the PTCD fistula. Another was delivered endoscopically to the obstruction of the jejunal side. The two magnets were immediately attracted towards each other transmurally, and reanastomosis was confirmed 7 days after starting the compression. The magnets were easily retrieved endoscopically. A 16-Fr indwelling drainage tube was placed in the jejunum through the PTCD. The internal tube is still in place 6 months after reanastomosis, and no MCA-related complications have been observed. In conclusion, MCA is a safe, effective, low-invasive treatment for biliary obstruction, and IDUS is useful for the pretreatment assessment of feasibility and safety.
\end{abstract}

Keywords: Magnetic compression anastomosis; Intraductal ultrasonography; Obstructed choledochojejunostomy

\section{Introduction}

Recently, magnetic compression anastomosis (MCA) was de-

\section{Manuscript submitted May 2, 2017, accepted August 3, 2017}

aDepartment of Gastroenterology, Kyoto Okamoto Memorial Hospital, $58 \mathrm{Ni}$ shinokuchi, Sayama, Kumiyama-cho, Kuze-gun, Kyoto 613-0034, Japan

${ }^{\mathrm{b}}$ Corresponding Author: Hideaki Kawabata, Department of Gastroenterology, Kyoto Okamoto Memorial Hospital, 58 Nishinokuchi, Sayama, Kumiyamacho, Kuze-gun, Kyoto 613-0034, Japan. Email: hkawabata@okamoto-hp.or.jp

doi: https://doi.org/10.14740/gr842w veloped by Yamanouchi et al $[1,2]$ as a non-surgical alternative treatment for obstruction of the common bile duct after choledochojejunostomy without serious complications [1-6].

However, the precise assessment of the local condition, such as that of vessels, inflammation and surgical suture threads, which may be related to complications and the outcome of MCA, is still difficult.

Intraductal ultrasonography (IDUS) provides real-time, high-quality, cross-sectional images of the extrahepatic bile duct and periductal structures [7]. Therefore, IDUS is generally performed during endoscopic retrograde cholangiography.

We herein report the case of obstructed choledochojejunostomy in which percutaneous IDUS proved useful for the local assessment before MCA.

\section{Case Report}

A 70-year-old woman underwent pancreaticoduodenectomy with modified Child reconstruction for pancreatic head cancer in March 2016. After the operation, the patient suffered anastomotic leakage at the site of gastrojejunostomy, and percutaneous intraperitoneal drainage (PID) was performed. The hepato-cholangio parameters such as total bilirubin, alkaline phosphatase, aspartate transaminase, and alanine aminotransferase levels, and computed tomography (CT) revealed an obstructed choledochojejunostomy with no recurrent findings 4 months after the operation. Percutaneous transhepatic cholangiography (PTC) confirmed complete obstruction of the upper common bile duct (CBD), which was the anastomosis of choledochojejunostomy, and percutaneous transhepatic cholangiographic drainage (PTCD) was performed. However, it was not able to pass the guidewire through the anastomosis. Cytology of the drainage from both PID and PTCD was negative for cancer.

After the recovery of the leakage and reducing ascites, enteroscopy (PCF-Q260AZI; Olympus Medical Systems, Tokyo, Japan) was performed. Although it was possible to reach adjacent to the anastomosis of choledochojejunostomy, the scar of the anastomosis was not observed. The length of the obstruction was $7 \mathrm{~mm}$, as measured by cholangiography and fluoroscopy (Fig. 1). IDUS (20 MHz, UM-3R, Olympus Medical Systems) through the PTCD tube dilated to $22 \mathrm{Fr}$ demonstrated a fibrous heterogeneous hyperechoic appearance; 


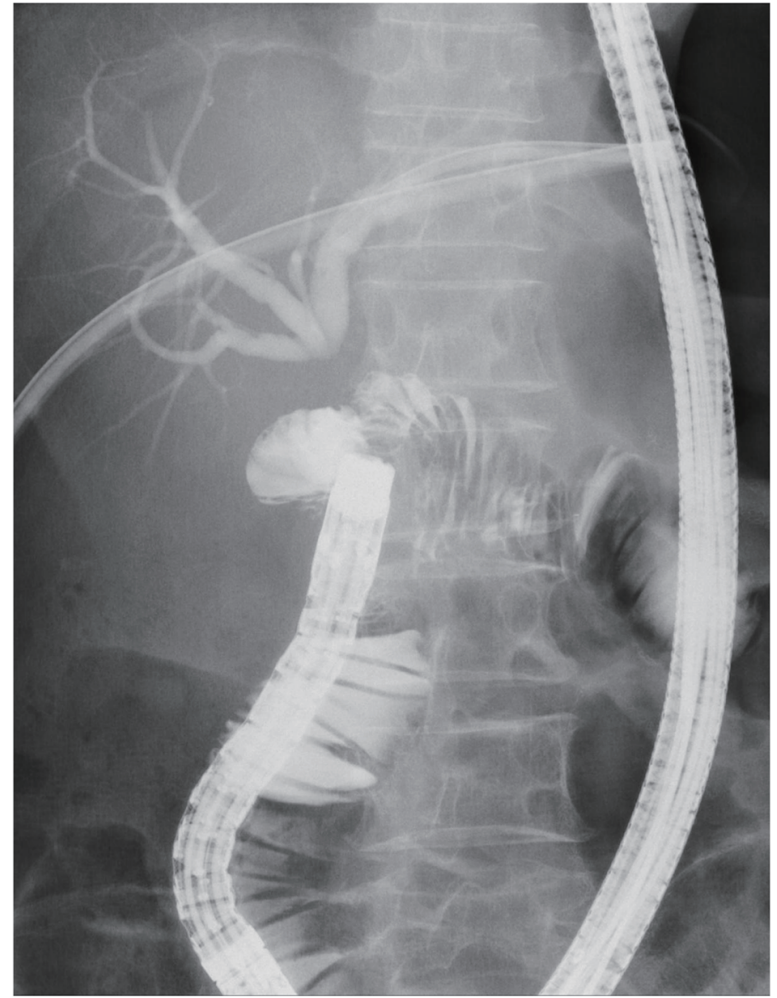

Figure 1. Percutaneous transhepatic cholangiography showed complete obstruction of choledochojejunostomy. The obstructive distance was $7 \mathrm{~mm}$, as measured by cholangiography and fluoroscopy.

however, no fluid collection, vessels or foreign bodies were observed between the CBD and jejunum (Fig. 2). We therefore performed choledochojejunostomy via the MCA technique. Written informed consent was obtained from the patient, and the institutional ethics review board at Kyoto Okamoto Memo-

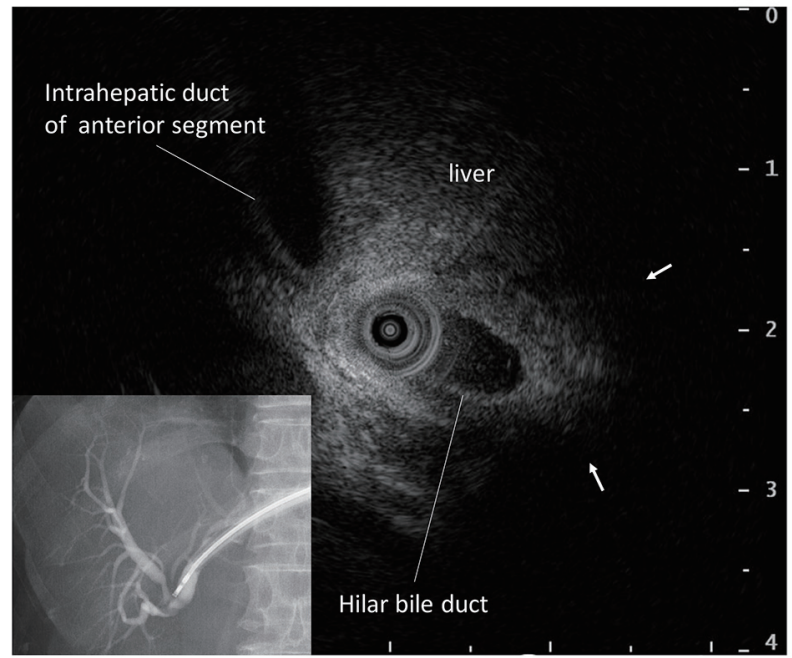

Figure 2. Intraductal ultrasonography through the percutaneous transhepatic cholangiographic drainage tube revealed a fibrous heterogeneous hyperechoic appearance without fluid collection, vessels or foreign bodies between the common bile duct and jejunum (arrow).

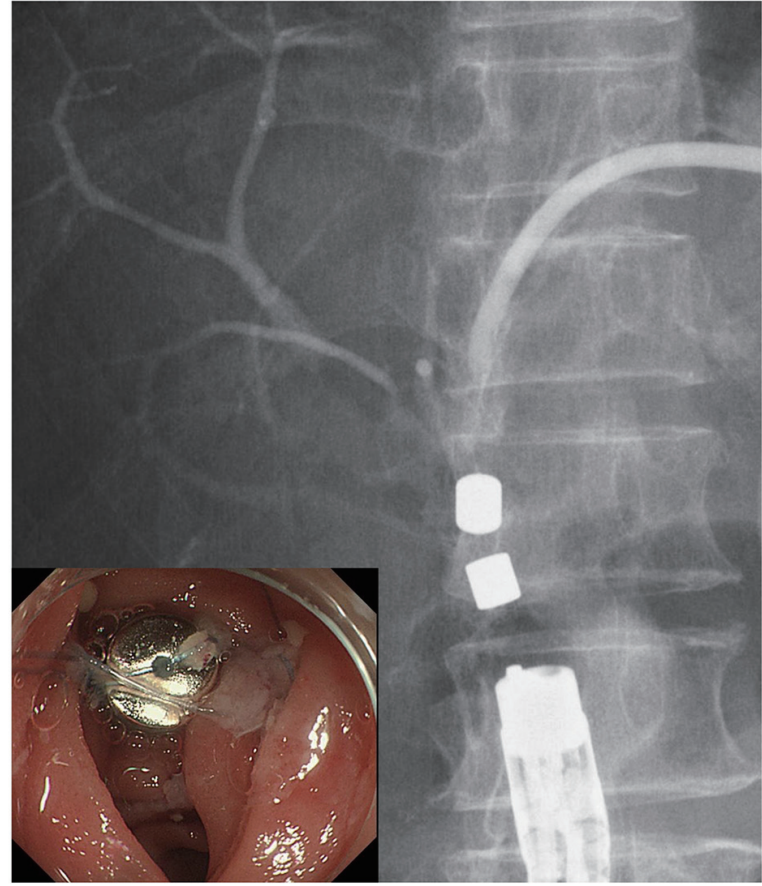

Figure 3. The two magnets were attracted towards each other transmurally and compressed the obstruction site from both sides.

rial Hospital approved this treatment.

We prepared two cylindrical neodymium magnets $(6 \times 6$ $\mathrm{mm}$; Magfine, Sendai, Japan) each with a small hole $1 \mathrm{~mm}$ in diameter, to which a nylon thread was passed through each hole. One magnet was inserted into the obstruction of the hepatic side through the PTCD fistula. The other was delivered endoscopically to the obstruction of the jejunal side. The two magnets were immediately attracted towards each other transmurally (Fig. 3). Reanastomosis was confirmed 7 days after starting the compression by cholangiography through the PTCD tube (Fig. 4). The magnets were easily retrieved endoscopically. A 16-Fr indwelling drainage tube was placed in the jejunum through PTCD tube to maintain the patency of the anastomosis (Fig. 5). The internal tube is still in place 6 months after reanastomosis and no MCA-related complications have been observed.

\section{Discussion}

Jang et al [4] reviewed 42 reported bilioenteric MCA procedures. All nine subjects with benign biliary obstruction developed complete anastomosis and no MCA-related complications. They also reported that recanalization was achieved successfully in 35 out of 39 patients $(89.7 \%$ ) who underwent MCA for completely obstructed benign biliary strictures, with the only procedure-related adverse event of mild cholangitis in one patient [5]. In addition, they suggested that the long distance between the two magnets, or non-linear alinement of the magnets often causes MCA failure. Furthermore, restenosis occurred in only three out of 39 follow-up patients after indwelling catheter removal. These findings in a relatively small 


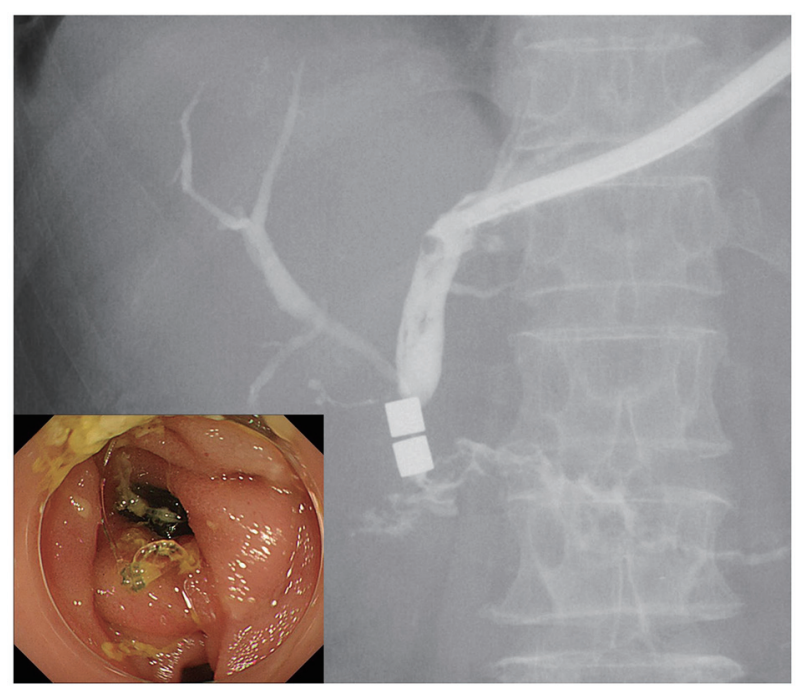

Figure 4. After reanastomosis was confirmed, the magnets were retrieved endoscopically.

number of cases imply that MCA is a feasible, safe alternative treatment for biliary obstruction. However, the major concern associated with this procedure is that a local assessment at the site of obstruction is difficult.

Blood vessels intervening between the two magnets may rupture, although no cases of blood vessel rupture have yet been reported, fortunately. In addition, foreign bodies such as suture threads or non-woven fabrics can cause the failure of reanastomosis [8]. Furthermore, effusion and thick fibrosis can lead to leakage and prevent fistula formation, respectively. Doppler ultrasound screening has been recommended to check the vascular flow $[2,9]$; however, a precise, accurate evaluation is difficult using the modalities available at present, such as ultrasonography, CT and magnetic resonance imaging. Contrast-enhanced endoscopic ultrasonography (EUS) with color Doppler is also used for the detection of major blood vessels between the magnets [2]; however, it is often difficult to insert an EUS endoscope due to the surgically altered gastrointestinal anatomy. In contrast to these modalities, IDUS is not only suitable for the local assessment within narrow limits, such as in MCA cases, but also can easily be performed through both the PTCD tube and endoscopic channels. In the present case, we confirmed fibrosis without vessels, foreign bodies or effusion at the site of obstruction by IDUS through the PTCD tube. Based on these pretreatment findings, we were able to estimate the feasibility and safety of the MCA procedure. To our knowledge, this is the first report showing IDUS to be useful for the pretreatment assessment of MCA.

However, IDUS for MCA has two limitations. First, the attenuation of the ultrasonic wave in the deep part of the obstruction may make the precise assessment difficult in cases of a long obstruction (longer than approximately $20 \mathrm{~mm}$ ). In those cases, IDUS through both the bile duct and intestine should be performed. Second, whether or not the obstructive site can be scanned by IDUS through the PTCD tube depends on the shape of biliary tract, although IDUS through endoscopy channel can be easily adjusted for scanning.

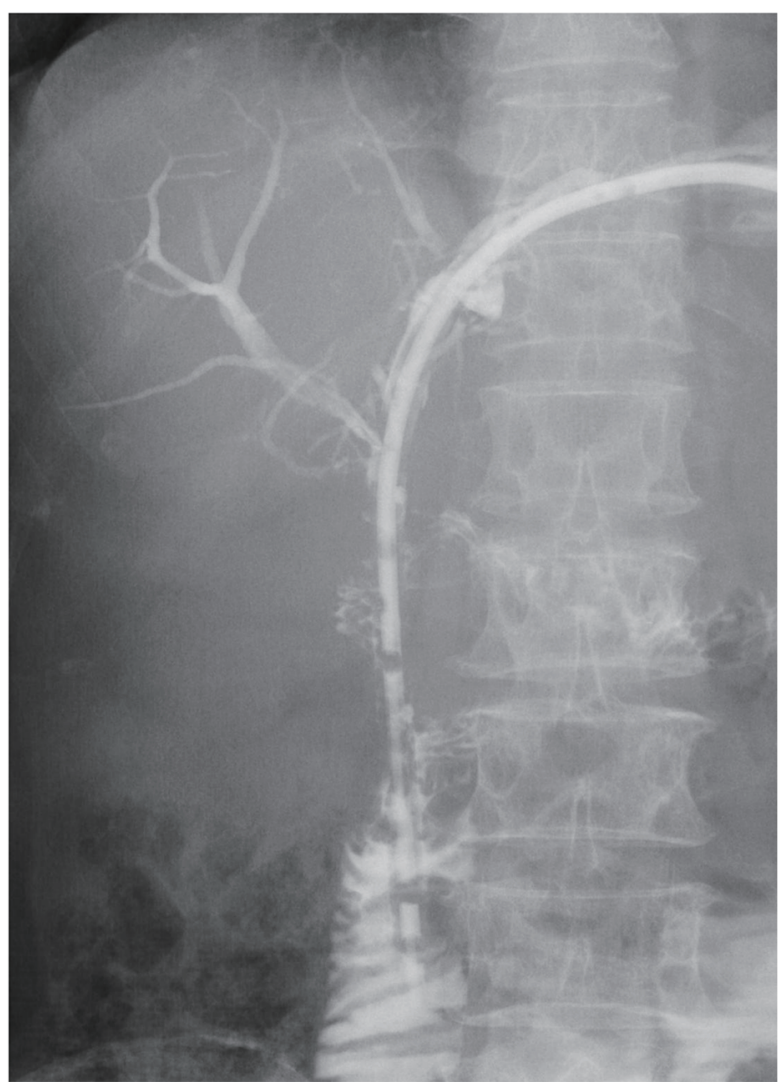

Figure 5. A 16-Fr indwelling drainage tube was placed in the juodenum through percutaneous transhepatic cholangiographic drainage tube.

In conclusion, MCA is a safe, effective, low-invasive treatment for biliary obstruction, and IDUS is useful for the pretreatment assessment of the feasibility and safety of MCA.

\section{Conflicts of Interest}

The authors declare that they have no conflicts of interest.

\section{References}

1. Takao S, Matsuo Y, Shinchi H, Nakajima S, Aikou T, Iseji T, Yamanouchi E. Magnetic compression anastomosis for benign obstruction of the common bile duct. Endoscopy. 2001;33(11):988-990.

2. Mimuro A, Tsuchida A, Yamanouchi E, Itoi T, Ozawa T, Ikeda T, Nakamura R, et al. A novel technique of magnetic compression anastomosis for severe biliary stenosis. Gastrointest Endosc. 2003;58(2):283-287.

3. Suyama K, Takamori H, Yamanouchi E, Tanaka H, Sakamoto Y, Ikuta Y, Maki Y, et al. Recanalization of obstructed choledochojejunostomy using the magnet compression anastomosis technique. Am J Gastroenterol. 2010;105(1):230-231.

4. Jang SI, Lee KH, Yoon HJ, Lee DK. Treatment of com- 
pletely obstructed benign biliary strictures with magnetic compression anastomosis: follow-up results after recanalization. Gastrointest Endosc. 2017;85(5):1057-1066.

5. Jang SI, Choi J, Lee DK. Magnetic compression anastomosis for treatment of benign biliary stricture. Dig Endosc. 2015;27(2):239-249.

6. Itoi T, Kasuya K, Sofuni A, Itokawa F, Tsuchiya T, Kurihara $\mathrm{T}$, Ikeuchi $\mathrm{N}$, et al. Magnetic compression anastomosis for biliary obstruction: review and experience at Tokyo Medical University Hospital. J Hepatobiliary Pancreat Sci. 2011;18(3):357-365.

7. Sun B, Hu B. The role of intraductal ultrasonogra- phy in pancreatobiliary diseases. Endosc Ultrasound. 2016;5(5):291-299.

8. Ito E, Ohdaira H, Nakashima K, Suzuki N, Imakita T, Tsutsui N, Yoshida M, et al. A case of transection-type bile leakage after laparoscopic cholecystectomy treated with magnetic compression anastomosis (Yamanouchi method). Prog Dig Endosc. 2015;87:80-83 (in Japanese).

9. Muraoka N, Uematsu H, Yamanouchi E, Kinoshita K, Takeda T, Ihara N, Matsunami H, et al. Yamanouchi magnetic compression anastomosis for bilioenteric anastomotic stricture after living-donor liver transplantation. J Vasc Interv Radiol. 2005;16(9):1263-1267. 